\title{
Design of Wideband Radio Direction Finder Based On Amplitude Comparison
}

\author{
Khalil Ibrahim Ali Al-Sharabi \\ College of Electronics Engineering \\ University of Mosul
}

\author{
Duraid Fiz'a Mohammad \\ College of Electronics Engineering \\ University of Mosul
}

\begin{abstract}
The design of the present radio direction finder is based on amplitude comparison to find the angle of arrival (AOA) of the received signal by eight antennas circularly distributed. The characteristics of the signal are specified to provide the identification of the source in addition to the direction. The equation of (AOA) was derived by taking the ratio of signal amplitudes between each two adjacent antennas due to their associated AOA over $\left(360^{\circ}\right)$ direction .The database for source identification is included also. A simple design by using detectors to detect the pulse and digital signals was used. A personal computer is used to satisfy the principle of operation. This facility make the system operate on a wide band of frequency about (218) GHz. This system is suitable for detection of radar signals and digital transmitter signals such as base stations and mobile stations.
\end{abstract}

Keywords: direction, finder, radio, frequency, amplitude, comparison

$$
\begin{aligned}
& \text { تصميم موجدة اتجاه راديوية واسعة النطاق بالأعتماد على مقارنة السعة } \\
& \text { دريد فزع محمد رضوان } \\
& \text { كلية هندسة الاكترونيات } \\
& \text { جامعة الموصل } \\
& \text { خليل ابر اهيمة علئي الثنر ابي }
\end{aligned}
$$

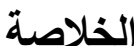

ان طريقة تصميم منظومة إيجاد الأتجاه في هذا البحث تعتمد على أسلوب مقارنة السعة للإثـارة المستلمة بين

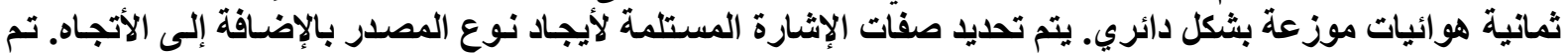

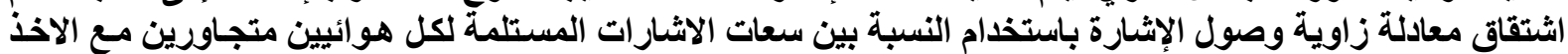

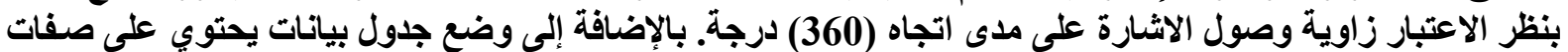

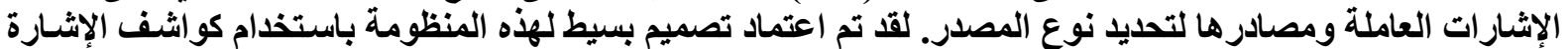

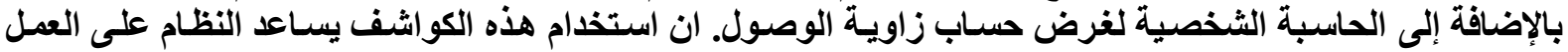

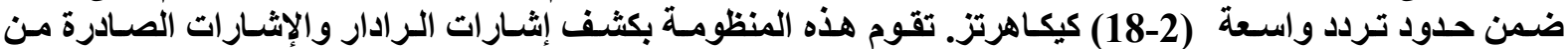

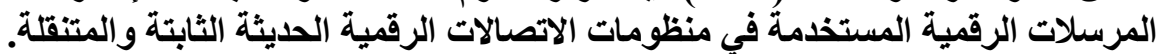




\section{Introduction}

Passive radio direction finders (DF) are used for finding the angle of arrival of the received signal over the whole $\left(360^{\circ}\right)$ direction [1]. This facility makes these systems important in military and civilian applications. There are many types of (DF's) depending on the principle of operation and the customer requirements and each type has its advantages and limitations. The conventional Adcock-Direction Finders suffer from system errors, matching problems, frequency limitations, and sighting [2, 3, 4]. The rotating antenna direction finders suffer from delay time to obtain the AOA because they need a long time to complete one cycle $\left(360^{\circ}\right)$ rotation (the speed of rotation is low). These systems require a pencil beam antenna and high precision mechanical system to provide accurate steering angle of antenna to represent the AOA which complicates its implementation [5]. The super heterodyne DF receivers require long time to scan the frequency over the designed range of frequency. Therefore they suffer from a delay time to find the AOA through one scan of frequency, therefore they are used for small range of frequency [6]. If a frequency scanner is used with direction finder then the system requires synchronization between frequency of the desired signal and measuring the AOA which need more measurement time. The phase difference DF's cannot cover the whole angle $\left(360^{\circ}\right)$ because they cover only a section of a determined angle and require a time and mechanical system to steer the antenna array to the other directions [7, 8]. The direction finders using the principles of amplitude comparison are mostly simple in design and implementation. This type of DF's can be made in different methods. Depending on design philosophy some of them use the difference between the logarithmic values of antenna gains [9], the others use the amplitude Sum-Difference method [10]. To simplify the amplitude comparison analysis, the ratio between signal levels which are fed from receiving antennas of the proposed DF is used in this paper [11]. This method makes the system simple in analysis and implementation than the previous methods, and gives good accuracy and resolution over a wide range of frequencies. The proposed system in this paper operates over a wide band of frequency and covers the whole $\left(360^{\circ}\right)$ by using frequency independent antennas circularly distributed as shown in fig. (1). In this system, detectors are used to detect the envelop signal of information to overcome the delay time required for scanning frequency over the whole band. This system provides the horizontal (azimuth) direction of the arrived signal as related to the reference direction automatically without steering antennas and then overcomes the mechanical problems.

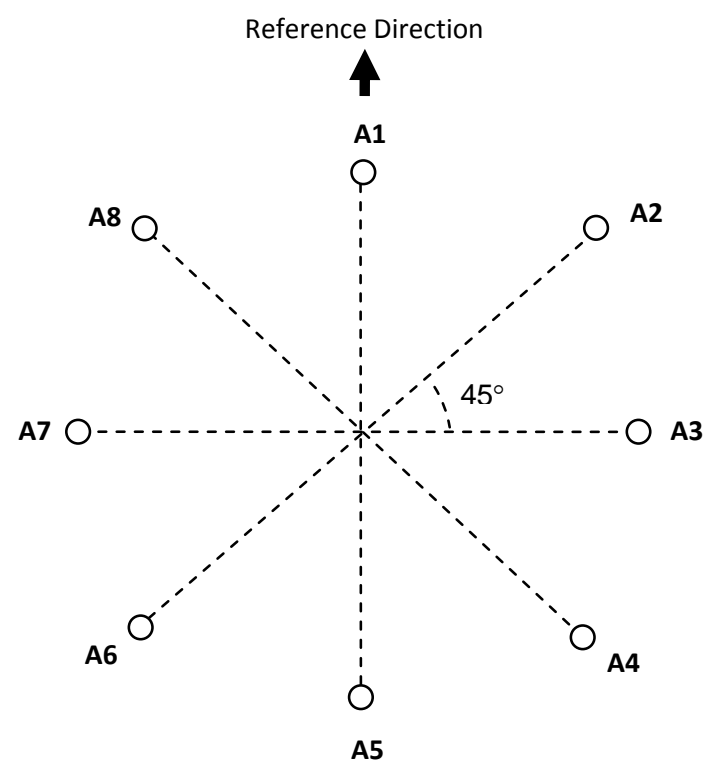

Figure (1) Distribution of Antennas 
In this system the amplitude comparison is applied between each two adjacent antenna to select the largest and next to the largest amplitude to provide the AOA of the received signal as will be explained later. The use of eight antennas will tend to reduce the angular error in measuring AOA [9]. This paper is devoted to describe an automatic DF system developed primarily for such use. There are many requirements that must be met if a system of automatic DF is to be accepted such as reliability, simplicity, sensitivity, light weight and accuracy. All ofthese factors must be considered in the system described here.

\section{Theory}

For starting derivation of AOA equation, it can be assumed that the antennas type is circular aperture with Gaussian current distribution and has Gaussian field patterns. The field pattern of the antenna assembly for the system is shown in fig. (2a). When a signal strikes the antenna assembly, the individual channel amplitude is measured as well as the difference between each two adjacent antennas to allow the selection of the channels having the largest and next to the largest amplitudes. To minimize the error of bearing (AOA) the antennas must be designed to have the suitable field pattern and the half power angle depending on the number of antennas used. By taking any two adjacent of antenna field patterns as shown in fig. (2b). It can be seen that the squint angle $(\alpha)$, which is the angle between the cross point of field patterns and the main lobe maximum direction lines (for eight antennas $2 \alpha=45$ ). Therefore the half power beam width $\left(\theta_{\mathrm{B}}\right)$ can be given by taking the general equation of the antenna has Gaussian field pattern as follow [9]:

$$
G_{A}=e^{-k^{2} \theta^{2} / \theta_{B}^{2}}
$$

Where $(\theta)$ is the angle of arrival with respect to the maximum gain point of the antenna, and $\left(k^{2}\right)$ is a factor that makes the value of $\left(G_{A}\right)$ equal to $(0.707)$ at angle $\left(\theta_{\mathrm{B}} / 2^{\circ}\right)$, so that $\left(k^{2}=\ln (4)=\right.$ 1.384). By taking the signal to noise ration

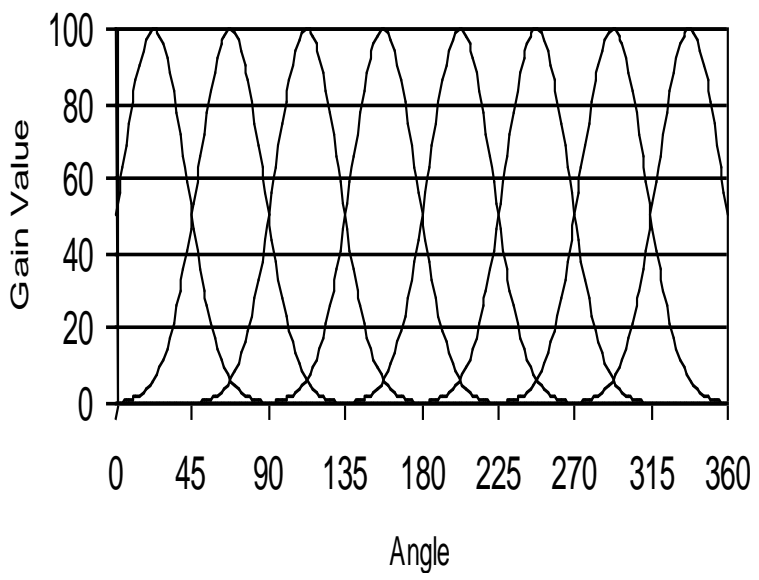

Figure (2a)

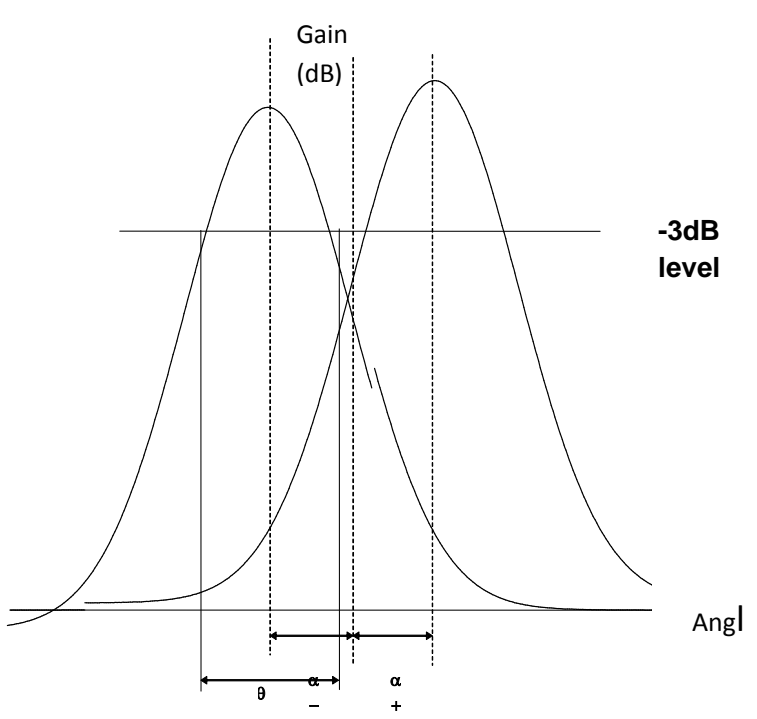

Figure (2b)

Figure (2) Antenna field patterns

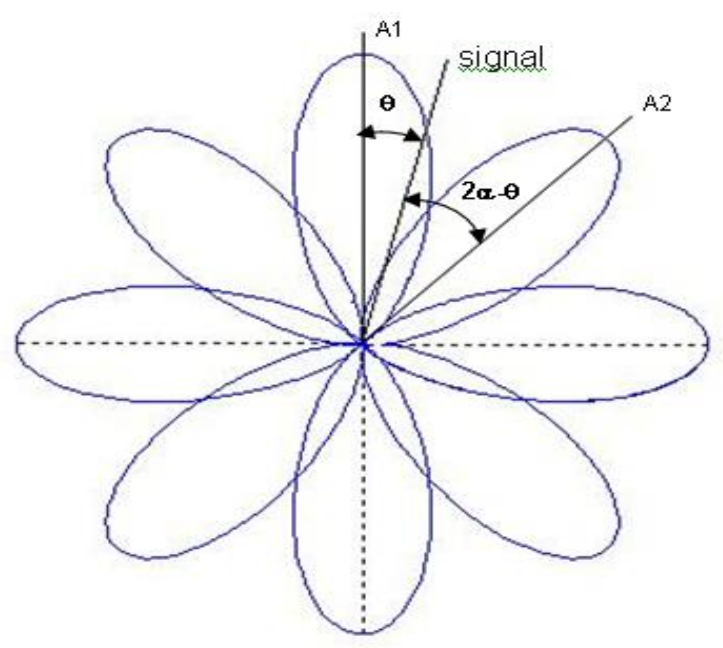

Figure (3) Polar Form Field Pattern with signal strikes system 
(SNR) into account for Gaussian field pattern antennas in the DF system, the optimum value of the squint angle $(\alpha)$ is given by Richard G. Wiley for $\mathrm{SNR}_{\max }$ assumed to be (14 dB) [9].

$\theta_{B}=2 \sqrt{\ln (2)} \alpha$

Then $\alpha=0.6 \theta_{\mathrm{B}}$

Since for eight antenna system $\alpha=22.5^{\circ}$ then $\theta_{\mathrm{B}}=37.5^{\circ}$

As an incident wave strikes the antenna assembly at angle $(\theta)$ from the maximum gain point of the reference antenna (A1) for which its broadside line coincides with the reference direction, the amplitude of the signal will be multiplied by the gain of each antenna relatively according to its associated angle of arrival with respect to that antenna. As an example if the signal arrives between antennas (A1) and (A2), the AOA will be less than $\left(45^{\circ}\right)$ as shown in fig. (3).

By taking the ratio $(\mathrm{R})$ between antenna gains at angle $\theta$ then:

$$
R=\frac{G_{A 1}}{G_{A 2}}
$$

Since the angle $(\theta)$ is the AOA of the signal from the maximum gain point of antenna (A1) and it lies between $\left(0^{\circ}\right.$ and $\left.45^{\circ}\right)$ then the AOA of the signal from antenna (A2) will be (45- $\theta$ ). In general for any number of antennas in the system this angle can be given as $(2 \alpha-\theta)$. Since this angle lies between antennas (A1 and A2) it is expected that the largest and next to the largest amplitude of the signal will be obtained from antennas (A1 and A2).

To derive the general equation of AOA, let us take the first two antennas A1 and A2, and generalize the equation for any number of antennas. If the carrier signal is sinusoidal of a function (M sin (wt)) where (M) is the maximum amplitude of the signal, ( $w=2 \pi f)$, and (f) is the operation frequency. The ratio between signal amplitudes from antenna (A1 and A2) will be:

$$
R=\frac{M 1}{M 2}
$$

Where M1 is the output of antenna A1 and it is given as:

$$
M 1=M \sin (w t) e^{-K^{2} \theta^{2} / \theta_{B}^{2}}
$$

And M2 is the output of antenna A2 and it is given as:

$$
M 2=M \sin (w t) e^{-K^{2}(45-\theta)^{2} / \theta_{B}^{2}}
$$

Substituting values of M1 and M2 in equation (4) then:

$$
R=\frac{e^{-k^{2} \theta^{2} / \theta_{B}^{2}}}{e^{-k^{2}(45-\theta)^{2} / \theta_{B}^{2}}}
$$

But for eight antennas $(2 \alpha=45)$ then equation (7) can be solved as:

$$
R=e^{-4 k^{2} \alpha(\theta-\alpha) / \theta_{B}^{2}}
$$

By solving equation (8), then $(\theta)$ will be given as: 
$-\theta=\frac{\theta_{B}^{2}}{4 k^{2} \alpha} \ln (R)-\alpha$

To generalize equation (9) for all antennas in the system to cover $\left(360^{\circ}\right)$ direction, let:

$\theta_{n}=\theta+45(n-1)$

Where $\mathrm{n}=1,2,3,4, \ldots ., 8$ is the number of antennas

For the system has any number of antennas (n), equation (10) can be written in the general form as:

$\theta_{n}=\theta+2 \alpha(n-1)$

and $\quad \alpha=360^{\circ} / 2 \mathrm{n}$.

In general for any system has any number of antennas, the general formula of AOA will be written as:

$\theta=\frac{\theta_{B}^{2}}{4 \alpha k^{2}} \ln (R)-\alpha+2 \alpha(n-1)$

\section{Angular Error}

For wide band DF's, it was found that the angular error in measurement of the angle of arrival of the signal varies with frequency due to the change of the half power beam width $\left(\theta_{\mathrm{B}}\right)$ over the frequency range and the signal to noise ratio. The antenna type proposed in this paper is assumed to have Gaussian field pattern, and small size to operate at high frequency range independently with small aperture [10]. For Gaussian distributed field pattern circular aperture antenna the half power beam width is given as $[12,13]$ :

$\theta_{B}=\frac{29.4 \lambda}{d}$

Where (d) is the dimension of antenna, and $(\lambda)$ is the wavelength associated with frequency of operation and

$\lambda=\mathrm{C} / \mathrm{f}$

where $C$ is the speed of light $\left(3 \times 10^{8} \mathrm{~m} / \mathrm{sec}\right)$.

The signal to noise ratio is also an important parameter for the angular error calculation which is a combination of the field noise near antenna and the thermal noise of the receiver [14]. The thermal noise is constant and depends on type of receiver components. But the field noise is due to the other signal noise and the interference between antennas due to effect of their side lobes. To calculate the maximum angular error, the maximum signal to noise ratio $\left(\mathrm{SNR}_{\max }\right)$ must be determined as the ratio of the received signal at the maximum gain point of an antenna and the noise level. In other words $\mathrm{SNR}=20 \log (\mathrm{M} / \mathrm{N})$, and $\mathrm{SNR}_{\max }$ $=20 \log \left(\mathrm{M}_{\max } / \mathrm{N}\right)$, where $\left(\mathrm{M}_{\max }\right)$ is the maximum level of signal at the maximum gain of antenna, and $(\mathrm{N})$ is the noise level [14]. As we assumed the $\mathrm{SNR}_{\max }$ equal to $(14 \mathrm{~dB})$ to 
satisfy the operation of the DF receiver with good accuracy and resolution, the angular error is given as [9]:

$$
\rho_{\theta}=\frac{20 \log e\left(2 / S N R_{\max }\right)^{1 / 2} \theta_{B}}{251}
$$

where $\rho_{\theta}$ is the angular error in degrees.

Figure (4) shows the variation of angular error with frequency from which it can be found that the maximum error in the angle of arrival is less than (2) degree.

From equation (14) it was seen that as the $\mathrm{SNR}_{\max }$ increases, the angular error will decrease. In practice the angular error can be measured experimentally by operating the system with an other reference direction finder in use and take the root mean square error value (RMSE) of many experiments (at least 100 number of experiments) to give a prediction of angular error which must be about (2) degrees.

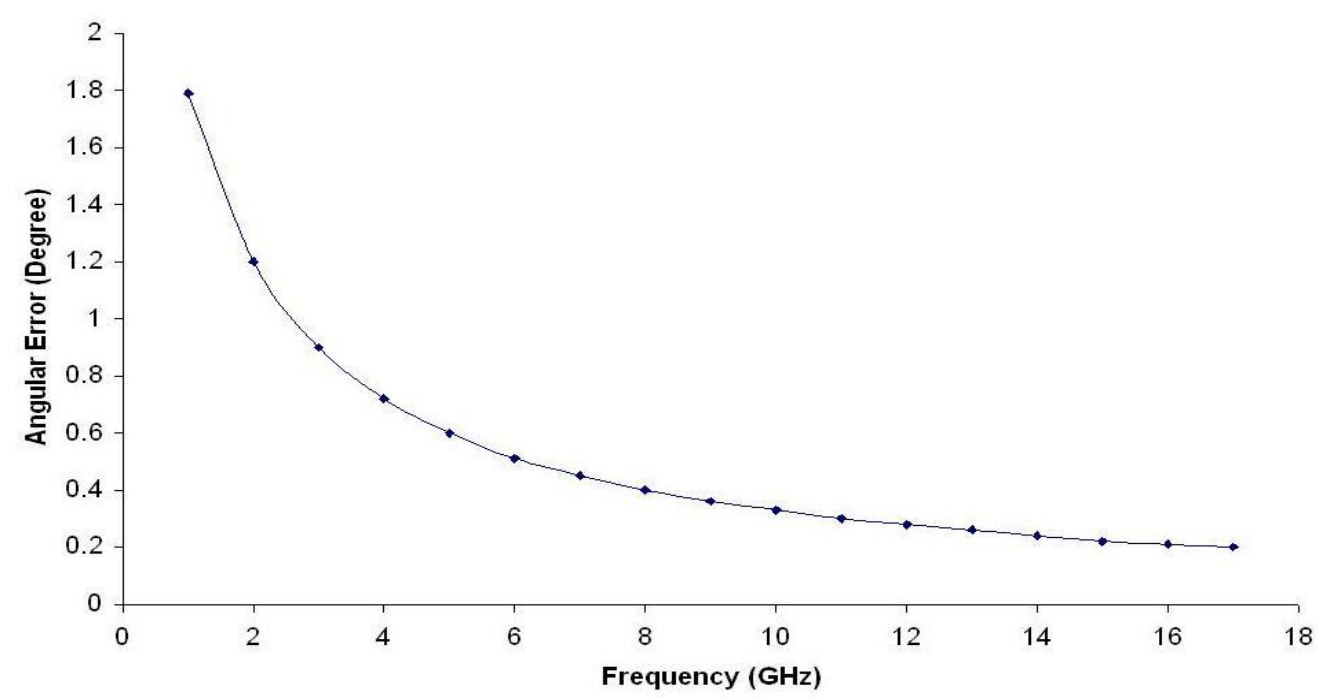

Figure (4) Variation of angular error versus frequency

\section{Programming and Results}

The performance of the direction finder is made by programming the personal computer (PC) and tested using $\mathrm{C}++$ programs to calculate the gain ratio between each two adjacent antennas applying equation (7) to calculate the AOA for eight elements circularly distributed. All signals received by antennas are assumed to be sinusoidal carriers with the same frequency and power. The ratios between the outputs of each two adjacent antennas are listed in Table (1) with an increment of angle (20) degrees ( for practical application this increment must be at least 0.1 degree for accurate results).

The performance of the DF can be verified by entering different data as an input for different signal levels received from each antenna and take the results representing the angle of arrival by applying equation (12). If there is unsatisfied entry of signal levels, a message will appear to show that there is a malfunction in the system. Some examples of signal condition with associated results are given in table (2) below. 
Table (1) Lock up table for gain ratio of each adjacent antennas versus angle

\begin{tabular}{|c|c|c|c|c|c|c|c|c|}
\hline Angle $\left({ }^{\circ}\right)$ & $\mathrm{R} 12$ & $\mathrm{R} 23$ & $\mathrm{R} 34$ & $\mathrm{R} 45$ & $\mathrm{R} 56$ & $\mathrm{R} 67$ & $\mathrm{R} 78$ & $\mathrm{R} 81$ \\
\hline 20.0 & 0.9546 & 0.0768 & 0.0001 & 0.0000 & 0.0000 & 0.0000 & 0.0000 & 0.2540 \\
\hline 40.0 & 0.8518 & 0.3784 & 0.0036 & 0.0000 & 0.0000 & 0.0000 & 0.0000 & 0.0411 \\
\hline 60.0 & 0.3558 & 0.8723 & 0.0458 & 0.0001 & 0.0000 & 0.0000 & 0.0000 & 0.0031 \\
\hline 80.0 & 0.0695 & 0.9411 & 0.2727 & 0.0017 & 0.0000 & 0.0000 & 0.0000 & 0.0000 \\
\hline 100.0 & 0.0064 & 0.4752 & 0.7601 & 0.0260 & 0.0000 & 0.0000 & 0.0000 & 0.0000 \\
\hline 120.0 & 0.0003 & 0.1123 & 0.9915 & 0.1875 & 0.0008 & 0.0000 & 0.0000 & 0.0000 \\
\hline 140.0 & 0.0000 & 0.0124 & 0.6053 & 0.6317 & 0.0141 & 0.0000 & 0.0000 & 0.0000 \\
\hline 160.0 & 0.0000 & 0.0006 & 0.1729 & 0.9962 & 0.1229 & 0.0003 & 0.0000 & 0.0000 \\
\hline 180.0 & 0.0000 & 0.0000 & 0.0231 & 0.7353 & 0.5007 & 0.0073 & 0.0000 & 0.0000 \\
\hline 200.0 & 0.0000 & 0.0000 & 0.0014 & 0.2540 & 0.9546 & 0.0768 & 0.0001 & 0.0000 \\
\hline 220.0 & 0.0000 & 0.0000 & 0.0000 & 0.0411 & 0.8518 & 0.3784 & 0.0036 & 0.0000 \\
\hline 240.0 & 0.0000 & 0.0000 & 0.0000 & 0.0031 & 0.3558 & 0.8723 & 0.0458 & 0.0001 \\
\hline 260.0 & 0.0000 & 0.0000 & 0.0000 & 0.0001 & 0.0695 & 0.9411 & 0.2727 & 0.0017 \\
\hline 280.0 & 0.0000 & 0.0000 & 0.0000 & 0.0000 & 0.0064 & 0.4752 & 0.7601 & 0.0260 \\
\hline 300.0 & 0.0008 & 0.0000 & 0.0000 & 0.0000 & 0.0003 & 0.1123 & 0.9915 & 0.1875 \\
\hline 320.0 & 0.0141 & 0.0000 & 0.0000 & 0.0000 & 0.0000 & 0.0124 & 0.6053 & 0.6317 \\
\hline 340.0 & 0.1229 & 0.0000 & 0.0000 & 0.0000 & 0.0000 & 0.0006 & 0.1729 & 0.9962 \\
\hline 360.0 & 0.5007 & 0.0000 & 0.0000 & 0.0000 & 0.0000 & 0.0000 & 0.0231 & 0.7353 \\
\hline
\end{tabular}

Table (2) Test results including calculated AOA for different signal levels

\begin{tabular}{|c|c|c|c|c|c|c|c|c|}
\hline A1 & A2 & A3 & A4 & A5 & A6 & A7 & A8 & AOA $\left(^{\circ}\right)$ \\
\hline 0.15 & 0.2 & 0.13 & 0.1 & 0.008 & 0.0 & 0.0 & 0.01 & -20 \\
\hline 0.4 & 0.1 & 0.0 & 0.0 & 0.0 & 0.01 & 0.02 & 0.5 & -32 \\
\hline 0.6 & 0.45 & 0.2 & 0.1 & 0.08 & 0.0 & 0.0 & 0.2 & -46 \\
\hline 0.1 & 0.2 & 0.65 & 0.5 & 0.08 & 0.0 & 0.0 & 0.0 & -136 \\
\hline 0.1 & 0.2 & 0.3 & 0.5 & 0.45 & 0.2 & 0.0 & 0.0 & -183 \\
\hline 0.0 & 0.0 & 0.04 & 0.08 & 0.55 & 0.65 & 0.1 & 0.0 & -231 \\
\hline 0.0 & 0.0 & 0.04 & 0.07 & 0.1 & 0.4 & 0.7 & 0.2 & -281 \\
\hline 0.2 & 0.05 & 0.0 & 0.0 & 0.0 & 0.1 & 0.5 & 0.6 & -317 \\
\hline 0.2 & 0.08 & 0.0 & 0.0 & 0.5 & 0.7 & 0.5 & 0.6 & Error \\
\hline
\end{tabular}

\section{Development of the System}

To simplify the development and implementation, a frequency independent receiver can be suggested by using wide band frequency independent antennas and detectors for detection of the pulse and digital signals as shown in fig. (4).

The antenna assembly consists of eight identical antennas circularly distributed, one of them is the reference antenna with its broadside coincides with reference direction. The type of antenna proposed to be used in such system must be frequency independent antenna such as Archimedean spiral, log periodic antennas that must be designed to operate over frequency band (2-18) GHz. taking into account the field pattern equation for any type of 
antenna used in the system design. The antenna must have $\left(\theta_{\mathrm{B}}\right)$ equal to $\left(37.5^{\circ}\right)$ half power beam width. The antenna has (2:1) SWR, and (1.1) axial ratio of polarization on bore sight over a (10:1) bandwidth [15]. The designer can select any type of antenna taking in consideration the field pattern equation for the calculation of beam width, squint angle, and AOA. The distance between any two adjacent antennas must be more than the longest

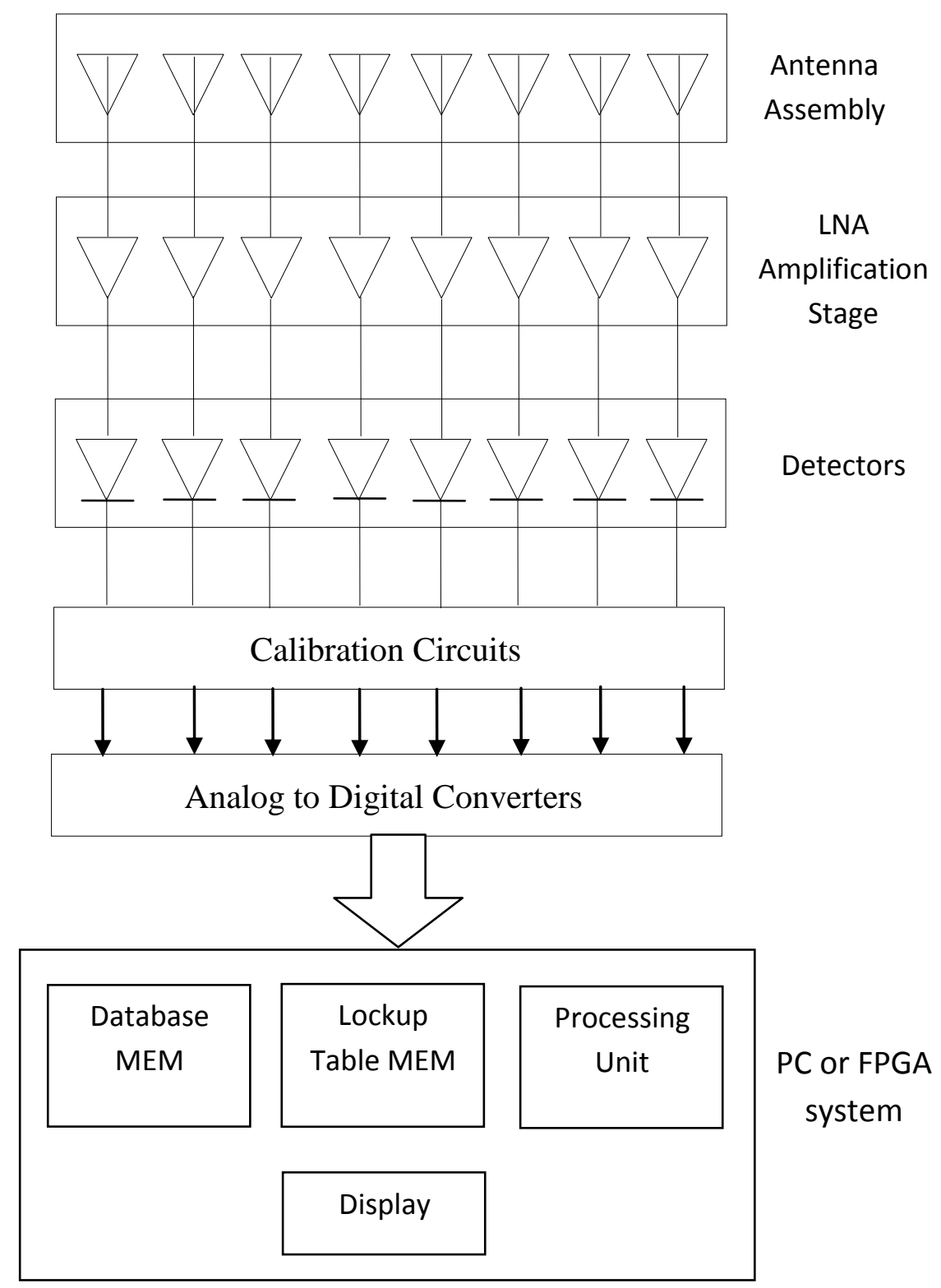

Figure (4) Basic Circuit Diagram of the Automatic Direction Finder System

wavelength at the lowest frequency of operation in order to minimize the interaction between antennas. Each antenna is followed by a Low Noise Amplifier (LNA) of (1.2) noise factor, and $(20 \mathrm{~dB})$ gain with high stability over the frequency band (2-18) GHz. For such system it is preferred to follow this amplifier by another stage of amplifier to increase the 
sensitivity. All amplifiers must be identical in all lines. Each amplifier is followed by a detector to detect the envelop signals. Since it is very difficult to get identical parts in all lines, calibration circuits must be used to calibrate these lines to make their responses identical. This calibration must be done during the experimental efforts. The analog to digital converter is used to convert the analog values of signals to digital forms to be fed to the personal computer (PC) which is used to calculate he angle of arrival of the signal. The PC has also two memories one is for database including the specifications of the signals and their associated sources to provide the source identification. The other is for the lockup tables including the pre calculated values of the gain for each antenna at their associated angles over $\left(360^{\circ}\right)$ direction depending on equation (1). The value of AOA which is calculated in the PC depending on equation (12) is compared with the AOA stored in the lockup table to give the status of results. The final part of the system is the console which displays results including signal specification, source identification, angle of arrival, and angular error.

\section{Conclusion}

There are many features of the system proposed in this paper, the main feature of the system is to compare the amplitudes between all antennas to provide the AOA of the incoming signal, that can make it suitable for military application such as Radar-WarningReceiver (RWR) which is used to detect the pulse radar signals, and for civilian application such as direction finders for digital communications (mobile systems). This system can be used also in smart antenna such as switched-beam smart antenna system [16] to provide Space Division Multiple Access (SDMA) for mobile communication. This system can be extended to receive the analog signals and can be developed to determine the frequency of the received signals by adding local oscillators to scan the frequency band or using a filter bank. The small size of antennas, detectors and PC or FPGA system make the system small in size, simple in use and implementation. The detectors will make the system frequency independent and can receive all signals in the space at the same time. The system does not require mechanical parts to steer antennas. It is realized that the system is built and operate to obtain sensitivity and accuracy comparable with other systems in use. The signal to noise ratio depends mostly in the field patterns of antennas therefore as the gain of antenna increases the $\mathrm{SNR}_{\max }$ will increase resulting of angular error decrease. For this reason it was concluded that as the number of antennas increases the angular error decreases. Finally it is concluded that as the number of antennas increases the angular error of AOA will decrease, and the system can operate with any type of directive antenna tacking into account the field pattern.

\section{References}

1. T. Matsumoto and Y. Kuwahara, "Experiments of Direction Finder by RBF Neural Network with post processing", Electronics Letters, $12^{\text {th }}$ May 2005, Vol. 41, No. 10.

2. J. D. Haigh, "Radio Location Techniques", 1960.

3. P. J. D. Gething, "Determination of Octantal Error on a 4-Elements ADOCK Direction Finder", Electronic Letters, Oct. 1966, Vol.2, No.10, pp. 388-389.

4. Y. T. Chan, B. H. Lee, Q. Yuan, "Direction Finding with a Four-Elements ADOCKButler Matrix Antenna Array", IEEE Transaction on Aerospace and Electronic Systems, Vol. 37, No. 4, Oct. 2001, pp. 1155-1162.

5. Charles H. Cotter, "The Principles and Practice of Radio Direction Finding", 1961. 
6. J.G. Worms, IMTC "COSBA an Experental System for Direction Finding and Signal Parameter Estimation", Instrumentation and Measurement Technology Conference, Como, Italy, 18-20 May 2004, pp. 869-872.

7. W. G. Diab, H. M. Elkamchouchi, "A Deterministic Real-Time DOA-Based Smart Antenna Processor", The $18^{\text {th }}$ Annual International Symposium On Personal, Indoor, and Mobile Radio Communications (PIMRC 07), 2007

8. Kravets, C. Morhart, E. Biebl, "Design of Radio Frequency Direction Finder for Automatic Applications", Proceeding of the $36^{\text {th }}$ European Microwave Conference, Sept. 2006, Manchester UK.

9. Richard G. Wiley, "Electronic Intelligence : The Interception of Radar Signals", 1985, Ch. 4, pp. 75-106.

10. V. A. Kryachko, "The Variant of Equation of Direction Finding Characteristic of the Amplitude Sum-Difference Direction Finder", Ukraine. International Conference on Antenna Theory and Techniques, 9-12 Sept. 2003. Scevastopel, Ukraine, pp. 376-377.

11. C.C. Pine, "A New Type of Automatic Radio Direction Finder", Proceeding IRE, Aug. 1945 , pp. 522-527.

12. Constantine A. Balanis, "Antenna Theory and Design", $2^{\text {nd }}$ Edition, 1997, Ch. 12, pp. 575-650.

13. Thomas A. Milligan, "Modern Antenna Design", 1985, Ch. 6, pp. 129-178.

14. Antonio Kalis, "Direction Finding in IEEE 802.11 Wireless Networks, IEEE Transaction on Instrumentation and Measurements, Vol. 51, No.5, Oct. 2002, pp. 940948.

15. W. L. Stutzman, G. A. Thiele, "Antenna Theory and Design", 1981, Ch. 6, pp. 260-303.

16. V.Jain, A.Gupta, D. P. Agrawal "On_Demand Medium Access in Multihop Wirless Networks with Multiple Beam Smart antenna", IEEE Transaction on Parallel and Distributed Systems, Vol. 19, No. 4, April 2008, pp. 489- 502. 